\title{
Rapid Identification of Mycobacterium tuberculosis and Non Tuberculous Mycobacterium Isolates from Pulmonary and Extra Pulmonary Samples using MGIT320 Liquid Culture System and MPT64 Antigen Test
}

\author{
Qursheed Sultana, Ajaz Hussain*, Mohammed Abdur Rab Ansari, \\ Mohd Khaleel and Maimoona Mustafa
}

Department of Microbiology, Deccan College of Medical Sciences, Hyderabad, India

*Corresponding author

\section{Keywords}

Mycobacterial growth indicator tube (MGIT), Mycobacterium tuberculosis complex (MTBc), Non-tubercular mycobacterium (NTM), MPT64 antigen test and multiple drug resistant (MDR)

Article Info

Accepted:

10 December 2018 Available Online: 10 Januarv 2019

\section{A B S T R A C T}

Tuberculosis (TB) is a major public health problem in India and a leading cause of death in adults, especially among the economically productive age group. Historically TB has been associated with significant morbidity and mortality and remains a major global health problem. The present study was initiated to determine the prevalence of Mycobacterium tuberculosis, Non Tuberculous Mycobacterium and its resistance to first line AntiTubercular drug from both pulmonary and extra pulmonary samples A total of 583 properly collected samples (226 pulmonary and 357 extra pulmonary) from patients with clinical/radiological suspicion of Tubercular infection were included in this study. All the samples were screened by Zeihl-Neelsen AFB microscopy, and subjected to liquid culture using Mycobacterium Growth Indicator Tube (MGIT-320). Positive cultures were differentiated into Mycobacterium tuberculosis complex (MTBc) or non-tubercular mycobacterium (NTM) by immunochromatography assay using MPT-64 antigen. Further it was followed by drug susceptibility testing of MTBc isolates thereby identifying multidrug resistant strains. Out of 583 samples, 141 strains were isolated on MGIT-320 (81 pulmonary, 60 Extrapulmonary) and the detection time was 15 days. Mycobacterium complex isolates were 116 and Nontuberculous Mycobacteria were 25. Among Mycobacterium tuberculosis complex isolates 92(56 pulmonary, 36 Extrapulmonary) were sensitive to all the drugs and 24(16 pulmonary, 8 Extrapulmonary) were resistant to one or more drugs. Multiple drug resistant (MDR) isolates were 7(6 pulmonary, 1 Extrapulmonary). MDR-TB is gradually increasing due to improper diagnosis and inadequate treatment. Differentiating mycobacterium as MTBc and NTM supported by sensitivity testing by using liquid culture has proved to be helpful in early decision for chemotherapy in MDR-TB patients.

\section{Introduction}

Tuberculosis (TB) is a major public health problem in India and a leading cause of death in adults, especially among the economically productive age group. Historically $\mathrm{TB}$ has been associated with significant morbidity and mortality and remains a major global health problem. India accounts for one- fifth of the global burden of TB. It is estimated that about 
$40 \%$ of Indian population is infected with TB bacillus.(1) The prevalence and mortality due to TB in India were estimated to be 249 and 26 respectively per100,000 population.(2)The importance of early diagnosis and correct etiological identification of pulmonary tuberculosis need not be over-emphasised, since treatment is different for Mycobacterium tuberculosis and atypical Mycobacteria (nontuberculous Mycobacteria, NTM). World Health Organization has given guidelines for low and medium income countries for use of liquid culture systems and drug sensitivity testing for tuberculosis work. (3) The emergence of anti- tubercular drug resistance is an increasing public health problem and TB control programmes in industrialized and developing countries alike. (4) Drug resistance arises due to improper and irrational use of anti-tubercular drugs (ATDs) in chemotherapy of drug-susceptible TB patients. This improper use is a result of a number of actions including administration of improper treatment regimens and failure to ensure that patients complete the whole course of treatment. Essentially, drug resistance indicates a weakness in TB control program in that area. A patient who develops active disease with a drug-resistant TB strain can transmit this form of $\mathrm{TB}$ to other individuals. Strategies used for the clinical management of patients infected with drugresistant Mycobacterium tuberculosis scomplex (MTBC) are different, therefore, prompt detection, isolation, and implementation of alternate anti-tubercular treatment regimens are necessary for suitable management (5) (6). Moreover, early detection of such cases is of utmost importance in preventing spread of resistant bugs in the community. Automated nonradiometric systems for accelerated isolation of Mycobacterium tuberculosis complex (MTBC), being expensive, are available only in selected centres in India and third-world countries. However, most laboratories still depend upon conventional techniques, thus resulting in an extended reporting time of 4-5 weeks. The MGIT is a liquid broth medium that is known to yield better recovery and faster growth of mycobacteria. In addition to Middlebrook 7H9 liquid media, the MGIT tube contains an oxygen-quenched fluorochrome. It detects oxygen consumption induced by growing micro-organisms (7). There are a few published reports on the evaluation of Bactec MGIT 960 on extrapulmonary samples. An innovative rapid kit, MPT64-ICT, to detect an established marker of MTBC, the MPT64 antigen, by immune chromatography test (ICT)developed by Japanese scientists(8) found universal acceptance due to its simplicity, accuracy and rapidity. (9) (10) (11) Indian reports on EPTB in general and the use of rapid kits for confirmation of MTBC in particular are few.

The present study was initiated to determine the prevalence of Mycobacterium tuberculosis, NonTuberculous Mycobacterium and its resistance to first line Anti-Tubercular drug from both pulmonary and extra pulmonary samples among patients attending a tertiary care hospital in Hyderabad.

\section{Materials and Methods}

\section{Study design}

The study was carried out in the clinical Microbiology laboratory of a tertiary care hospital in Hyderabad during the period January 2013 to December 2015. Our Institutional Human Ethics Committee scrutinized and approved this research. Patients' informed consent was obtained before collection of specimens.

\section{Study population}

A total of 583 properly collected samples (226 pulmonary and 357 extra pulmonary) from patients with clinical/radiological suspicion of 
Tubercular infection were included in this study. We included both pulmonary (like deeply expectorated freshly collected sputum samples, free of saliva, blood and food contamination and bronchial alveolar lavage samples) and extra-pulmonary samples (such as all body fluids, tissue, urine, pus, aspirates etc.). Samples were included irrespective of the treatment status of the patients (e.g. both new suspected cases as well as post-treatment cases).

Patients were finally included on the basis of availability of consent forms. Any patient without consent was excluded from the study. All samples showing evidence of contamination with saliva (determined by Bartlett's grading system) (12) were excluded from our study. We excluded the whole blood samples as well as swab samples for TB diagnosis in this study as per standard guidelines.

\section{Inclusion criteria}

Both pulmonary (like deeply expectorated freshly collected sputum samples and bronchial alveolar lavage samples) and extrapulmonary samples (such as all body fluids, tissue, urine, pus, aspirates etc.) were included. All samples were selected on the basis of availability of consent.

\section{Exclusion criteria}

Swabs, Blood, salivary samples were excluded from our study.

\section{Materials and Methods}

\section{Acid fast bacilli smears}

Smears were prepared from each sample, stained by Ziehl Neelson method and examined for presence of AFB with a light microscope.
Decontamination and processing of the samples

All specimens were liquefied and decontaminated by the standard $\mathrm{N}$-acetyl-Lcysteine, sodium hydroxide method $(\mathrm{NaOH}-$ NALC). After 15 min holding at room temperature, specimens were neutralized with phosphate buffer saline (PBS, pH 6.8) and centrifuged in cold centrifuge at $4500 \mathrm{rpm}$ for $20 \mathrm{~min}$ at $10^{\circ} \mathrm{C}$. The pellets were resuspended in $1.5 \mathrm{ml}$ of sterile phosphate buffer and collected for further analysis.

\section{BACTEC MGIT 320 liquid media}

The BBL MGIT tube was inoculated by 0.5 $\mathrm{ml}$ of the decontaminated and concentrated specimen suspension. It contained $7 \mathrm{~mL}$ of modified middlebrook $7 \mathrm{H} 9$ broth enrichment with albumin, dextrose and catalase (BBL MGIT OADC) and an antibiotic mixture consisting of polymyxin $\mathrm{B}$, amphotericin $\mathrm{B}$, nalidixic acid, trimethoprim, and azlocillin (BBL MGIT PANTA). After inoculation, the tubes were loaded in the BACTEC MGIT 320 instrument and incubated up to 42 days at $37^{\circ} \mathrm{C}$. Culture vials are monitored hourly by the instrument. The positive tube was further confirmed by ZN staining, subculturing on blood agar plate. The TTD (Time to Detection) of mycobacteria was based on the date of the earliest instrumental indication of positivity.

\section{Morphological and biochemical identification}

For differentiation of M. tuberculosis complex and NTM, a commercially available kit was used, the BD MGIT MTBc identification test (TBc ID). It is a rapid chromatographic immunoassay for the qualitative detection of M. tuberculosis complex antigen from AFB smear-positive BD MGIT tubes. The assay is performed 
according to the manufacturer's instructions. Briefly, $100 \mu \mathrm{l}$ of mixed and vortexed culture fluid from AFB positive MGIT tubes were transferred to sample window of the cassette. The results of ICT were read within 15 minutes. Positive test had two red to purple bands, one for internal control and the secondline for the test.

Negative had only one band in internal control slot. Strong or light bands with any intensity were considered to be positive. MGIT tubes showing non-acid fast bacilli and/or fungi were excluded from MPT64 Ag test.

\section{BACTEC MGIT 320 liquid media DST}

MTBc isolates was further tested for the first line drugs in BACTEC MGIT 320. Conc. of various drugs used was - streptomycin (STR)- $1 \mu \mathrm{g} / \mathrm{ml}$, isoniazid (INH)- $0.1 \mu \mathrm{g} / \mathrm{ml}$, rifampicin (RIF)- $1 \mu \mathrm{g} / \mathrm{ml}$, ethambutol (ETB)$5 \mu \mathrm{g} / \mathrm{ml}$. Drug susceptibility was reported when the growth control units reached 400 as indicated by the instrument.

\section{Control strains}

Reference strains of H37Rv and Mycobacterium fortuitum were included as positive and negative controls, respectively.

\section{Results and Discussion}

583 clinical samples (226 pulmonary and 357 extra-pulmonary) were analyzed during the period of our study (Figure 1).

Number of Positive and Negative samples, screened through Ziehl-Neelsen AFB Staining procedure and culture by liquid media MGIT 320 are given in Table1. Out of these 583 samples, 257 were male patient and 326 were females, in which 63 and 78 were positive respectively, summarized in Table 2 . There was no much difference in gender distribution among positive pulmonary samples whereas females were predominant in case of Extra pulmonary positive samples (Figure 2 and 3 ).

The results of age wise distribution among positive cases in both pulmonary and Extra pulmonary samples shows majority of case in the age group of below 40 years, summarized in Table 3. Distribution of various samples is given in the Table 4.

The results show that, out of 226 pulmonary samples, 81 were MGIT culture positive, of which 47 were positive for AFB by $\mathrm{ZN}$ staining and out of 357 Extra pulmonary samples, 60 were MGIT culture positive, of which 19 were positive for $\mathrm{AFB}$ by $\mathrm{ZN}$ staining (Table 5 and 6). Out of these 81 culture positive isolates from pulmonary samples 71 were MPT64Ag test positive and 9 were negative samples. This was considered as Non-Tubercular Mycobacterium sp. (Speciation not done).

Similarly, Out of these 60 culture positive isolates from Extra pulmonary samples 44 were MPT64Ag test positive and 16 were negative samples. This was considered as Non-Tubercular Mycobacterium sp. (Speciation not done) (Table 7).

The average TTD was 15 days for MGIT 320 with the extremity from 6 to 38 days. Among pulmonary positive cases, resistance to any drug was found in 16 cases $(19.75 \%)$, to $\mathrm{S}$ in $5(6.17 \%)$, to $\mathrm{I}$ in $13(16.04 \%)$, to $\mathrm{R}$ in $7(8.64 \%)$ and to $\mathrm{E}$ in $2(2.46 \%)$. Multidrug resistance rate was6 $(7.40 \%)$ (Figure 4$)$.

Similarly among Extra pulmonary positive cases, resistance to any drug was found in 8 cases $(13.3 \%)$, to $\mathrm{I}$ in $2(3.33 \%)$, to $\mathrm{R}$ in $3(5.00 \%)$ and to $\mathrm{E}$ in $1(1.66 \%)$ and no mono resistance in $\mathrm{S}$. Multidrug resistance rate was1(1.66\%) (Figure 5). 
Table.1 Distribution of culture positive cases

\begin{tabular}{|c|c|c|}
\hline No. of cases studied & $\begin{array}{c}\text { No. of positive } \\
\text { cases }\end{array}$ & $\begin{array}{c}\text { No. of negative } \\
\text { cases }\end{array}$ \\
\hline 583 & $141(24.18 \%)$ & $442(75.18 \%)$ \\
\hline
\end{tabular}

Table. 2 Gender distribution of patients and percentage of positive samples

\begin{tabular}{|c|c|c|}
\hline Gender & $\begin{array}{c}\text { No. Of collected } \\
\text { samples }(\boldsymbol{\%})\end{array}$ & $\begin{array}{c}\text { Positive isolates } \\
(\boldsymbol{\%})\end{array}$ \\
\hline Male & $257(44.08)$ & $63(10.80)$ \\
\hline Female & $326(55.92)$ & $78(13.38)$ \\
\hline Total & 583 & 141 \\
\hline
\end{tabular}

Table.3 Age and Sex distribution of positive cases

\begin{tabular}{|c|c|c|c|c|}
\hline \multirow{2}{*}{$\begin{array}{c}\text { Age } \\
\text { Distribution }\end{array}$} & \multicolumn{2}{|c|}{ Pulmonary } & \multicolumn{2}{c|}{ Extra Pulmonary } \\
\cline { 2 - 5 } Male(n=41) & Female(n=40) & Male(n=22) & Female(n=38) \\
\hline $\mathbf{2 0}$ and below & $9(22 \%)$ & $13(32 \%)$ & $6(27 \%)$ & $8(21 \%)$ \\
\hline $\mathbf{2 1}-\mathbf{4 0}$ & $11(27 \%)$ & $16(40 \%)$ & $9(41 \%)$ & $13(34 \%)$ \\
\hline $\mathbf{4 1 - 6 0}$ & $15(36 \%)$ & $8(20 \%)$ & $4(18 \%)$ & $17(45 \%)$ \\
\hline $\mathbf{6 1}$ and above & $6(15 \%)$ & $3(8 \%)$ & $3(14 \%)$ & 0 \\
\hline
\end{tabular}

Table.4 Sample distribution in patients

\begin{tabular}{|c|c|c|c|}
\hline \multicolumn{2}{|l|}{ Type of sample } & No of cases & Positive \\
\hline \multirow{2}{*}{$\begin{array}{l}\text { Pulmonary } \\
n=226(38.77 \%)\end{array}$} & Sputum & 195 & 76 \\
\hline & BAL & 31 & 5 \\
\hline \multirow{11}{*}{$\begin{array}{l}\text { Extra pulmonary } \\
\mathrm{n}=357(61.23 \%)\end{array}$} & Pleural fluid & 199 & 27 \\
\hline & Pus & 41 & 11 \\
\hline & Ascitic fluid & 29 & 3 \\
\hline & ET Secretion & 11 & 4 \\
\hline & CSF & 19 & 2 \\
\hline & Urine & 8 & 1 \\
\hline & Pericardial fluid & 7 & 0 \\
\hline & Lymph Node Aspirates & 31 & 12 \\
\hline & Peritoneal fluid & 3 & 0 \\
\hline & Semen & 1 & 0 \\
\hline & Synovial fluid & 8 & 0 \\
\hline Total & & 583 & 141 \\
\hline
\end{tabular}


Table.5 Distribution of positive cases sample wise

\begin{tabular}{|l|c|c|c|}
\hline Sample Type & Total Samples & AFB Culture Positive & AFB Culture Negative \\
\hline Pulmonary & 226 & 81 & 145 \\
\hline $\begin{array}{l}\text { Extra } \\
\text { pulmonary }\end{array}$ & 357 & 60 & 297 \\
\hline
\end{tabular}

Table.6 correlation between stain and culture

\begin{tabular}{|l|c|c|c|c|c|c|}
\hline & \multicolumn{3}{|l|}{ Pulmonary } & \multicolumn{3}{l|}{ Extra Pulmonary } \\
\cline { 2 - 7 } & Stain +ve & Stain -ve & Total & Stain +ve & Stain -ve & Total \\
\hline Culture Positive & 47 & 34 & 81 & 19 & 41 & 60 \\
\hline Culture Negative & 0 & 145 & 145 & 0 & 297 & 297 \\
\hline
\end{tabular}

Table.7 MTBC and NTM positive samples

\begin{tabular}{|l|l|l|}
\hline Sample Type & MTBC & NTM \\
\hline Pulmonary & 72 & 9 \\
\hline Extra Pulmonary & 44 & 16 \\
\hline Total & $\mathbf{1 1 6}$ & $\mathbf{2 5}$ \\
\hline
\end{tabular}

Fig 1 Distribution of sample type

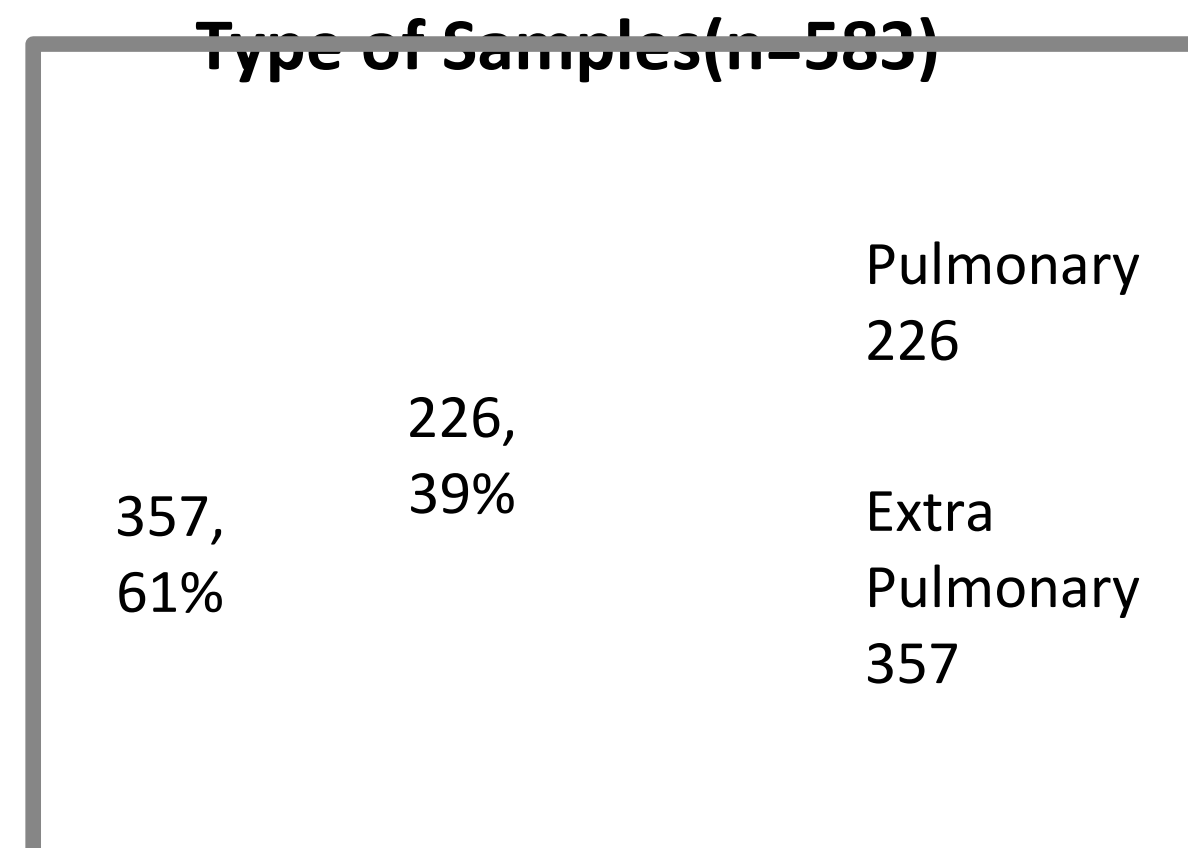


Fig.2 Sex wise distribution of MTB positive pulmonary samples

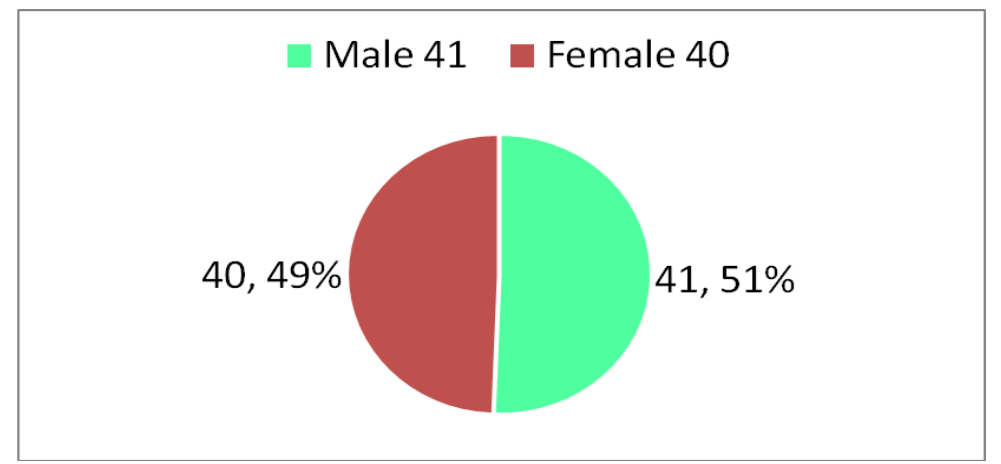

Fig.3 Sex wise distribution of MTB positive extra pulmonary samples

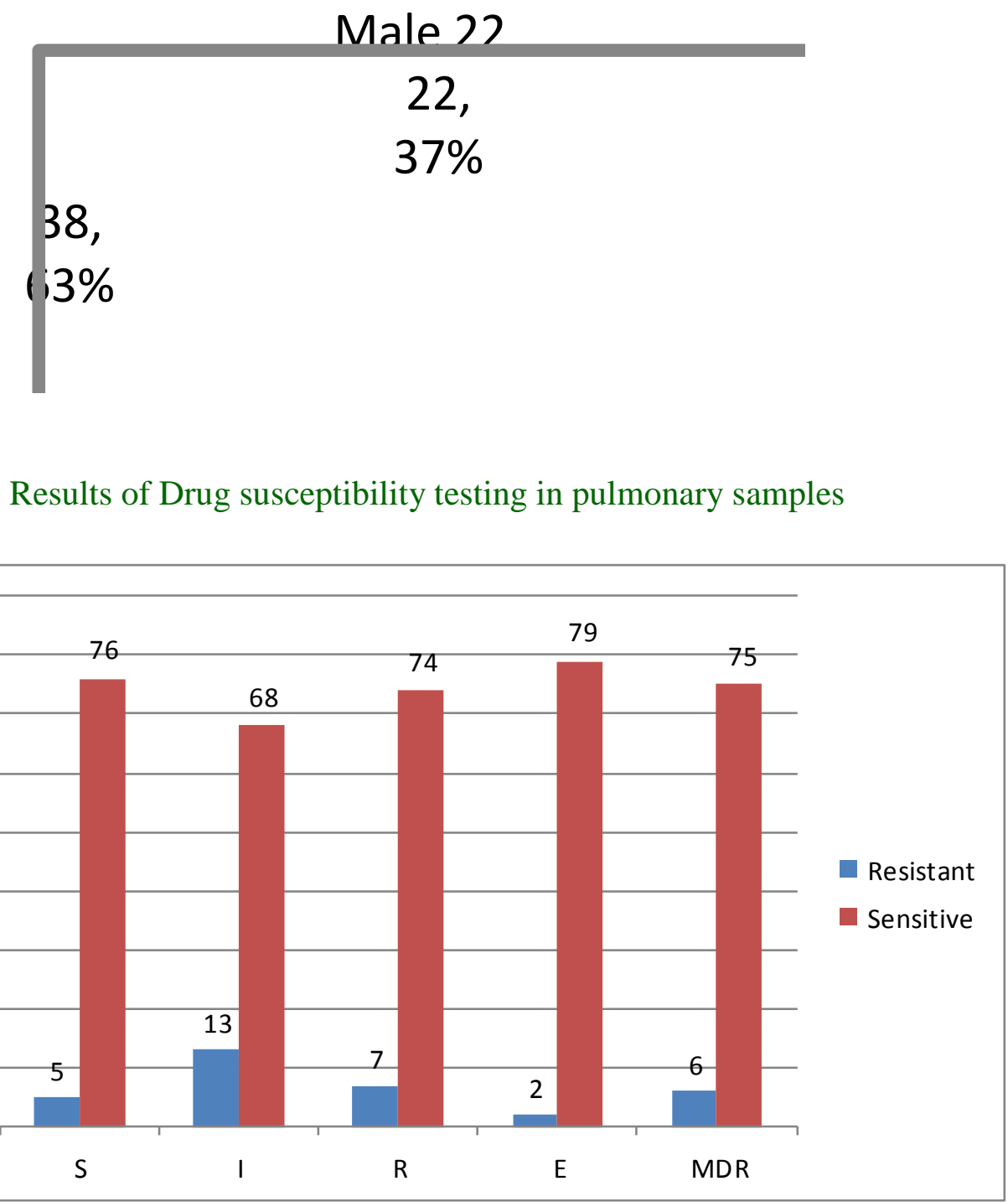


Fig.4 Results of Drug susceptibility testing in extrapulmonary samples

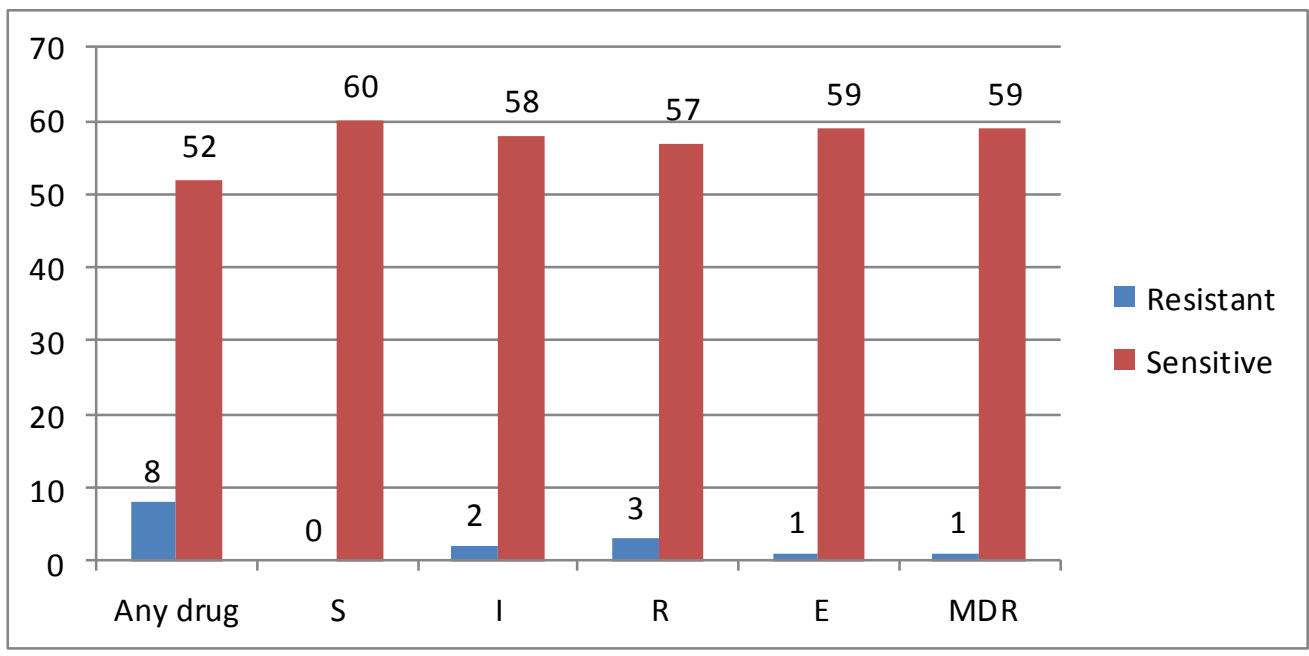

Early diagnosis of Mycobacterium tuberculosis infection is pre-requisite to achieve WHO's target to end Global TB epidemic. A definitive diagnosis of TB can only be made by culturing Mycobacterium tuberculosis organisms from a specimen obtained from the patient. Therefore, techniques which shorten the time for detection of Mycobacterium deserve attention.

In our study, out of the 583 clinical samples (both pulmonary and extra pulmonary), $141(24.18 \%)$ were culture positive. The importance of early diagnosis and correct etiological identification of Tuberculosis need not be over-emphasised, since treatment is different for Mycobacterium tuberculosis and atypical Mycobacteria (non-tuberculous Mycobacteria, NTM). In our study, out of 141 positive isolates $116(82.2 \%)$ were Mycobacterium tuberculosis (MTBc) and $25(17.7 \%)$ isolates were NonTuberculous Mycobacterium (NTM) using MPT64Ag test. Similar results were given in various studies like Kannade et al., (13) from Bombay (Mumbai) who examined 165 isolates (125 MTB; 30 NTM; 10 Non-Mycobacterial species) and observed sensitivity of $99.19 \%$ and $100 \%$ values for specificity, positive predictive value (PPV) and negative predictive value (NPV) for the rapid MPT64 antigen detection kits in comparison to conventional methods. Vadwai et al., (14) from Bombay analysed 394 strains from 280 pulmonary and 114EPTB samples (388 MTB; 6 NTM) with similar result, i.e. 99.4\% sensitivity and $100 \%$ specificity. Kumar et al., (15) from Mysore, Karnataka, analysed 77 isolates (55 MTB; 10 NTM; 12 NonMycobacterial species) recorded $100 \%$ results for all four parameters.

In our study majority of the pulmonary MTB infected male patients were within the age group of 20-40 years and female patients, within the age group of 10-40 years. In the case of extra pulmonary samples too both the males and females were from the age group of 20 - 40 years. This is in correlation with the study done by Kandhakumari et al., (16).

The prevalence of drug-resistant $\mathrm{TB}$ was found variable in different studies from around the world and in our country. In our study, out of the 583 clinical samples, among pulmonary samples the prevalence of resistance to any drug was found in 16 cases $(19.75 \%)$, to $\mathrm{S}$ in $5(6.17 \%)$, to $\mathrm{I}$ in $13(16.04 \%)$, to $\mathrm{R}$ in $7(8.64 \%)$ and to $\mathrm{E}$ in $2(2.46 \%)$. Multidrug resistance rate was6 
(7.40\%). Similarly among Extra pulmonary positive cases, resistance to any drug was found in 8 cases $(13.3 \%)$, to I in $2(3.33 \%)$, to $\mathrm{R}$ in $3(5.00 \%)$ and to $\mathrm{E}$ in $1(1.66 \%)$ and no mono resistance in S. Multidrug resistance rate was $1(1.66 \%)$. Multidrug-resistance is the independent factor for morbidity and mortality due to tuberculosis (17) (18). Treatment of MDR-TB is difficult and drugs used for treatment are less potent, more toxic and more expensive than firstline drugs (18) (20). Many studies published from different parts of India have reported high MDR-TB prevalence, but mostly among first-time retreatment patients with relapse, treatment after default, and treatment after failure (21) (22). The possible reasons of a higher prevalence of drug resistance in our study can be, mixing of new as well as retreatment cases and smaller sample size. Although many Indian studies have reported lower prevalence of Rifampicin mono-resistance from various parts of the country, in our study the higher rate can be due to a possible co-existence of INH resistance and the rate may be acting as a proxy to the local MDR-TB prevalence. Various Indian studies have reported MDR rates to be varying from $17.4 \%$ to $53 \%$ among re-treatment cases. $(23,24) \quad$ World-wide surveillance of MDR in re-treatment cases ranged from $9.4 \%$ to $36.5 \%$, from $1994-2000$ across the world.(25) Previous exposure to anti-tuberculosis agents is the most common cause of developing MDR. In 2008, the WHO reported a worldwide resistance rate to $\mathrm{INH}$ of $5.9 \%$. INH resistance rates higher than $10 \%$ can predict the development of MDR TB according to the WHO (26). The higher resistance rate of INH according to other first line drugs may be resulted by both its wide use in the chemoprophylaxis and latent TB (27).

According to $\mathrm{WHO}$ in 2014, 220,000 people died from TB in India, which is the highest in the world. The same report says that $2.1 \%$ cases in this emerging percentage are due to MDR-TB.

Thus early detection of MDR-TB cases and initiation of appropriate treatment based on drug resistance testing can lower the burden of this deadly disease.

In conclusion to conclude, globally the prevalence of Tuberculosis is on the increase. Due to prolonged time taken for positive culture and drug susceptibility report by conventional methods in suspected cases, the clinicians in developing countries empirically initiate anti-tuberculosis treatment (ATT) with first-line drugs. However, if the etiology happens to be NTM, this would be a burden to the patients and can promote emergence of drug resistance in Mycobacteria. The isolates must be checked for drug sensitivity in this era of increasing drug resistance. Thus rapid isolation of Mycobacterium species using automated MGIT320 system is more beneficial when combined with rapid ICT kit which detects MPT64 Ag in 15 minutes and also differentiates MTBC from NTM isolates. Notification of the DST results with clinical data is a key element to get valid and representative information on drug resistance. As a study of prevalence of drug resistance in TB from Hyderabad, we believe that this study can help in the control of TB at the national level and probably can help us in the mapping drug resistant $\mathrm{TB}$ cases in this part of the country.

\section{References}

1. Central Tuberculosis Division. Revised National TB Control Programme: Annual Status Report 2011. Available at: Http:// tbcindia.nic.in/pdfs/RNTCP\%20TB\%20In dia\%202011.pdf

2. Central Tuberculosis Division. Revised National TB Control Programme: Annual Status Report 2013. Availableat: 
Http://www.tbcindia.nic.in/pdfs/tb\%20indi a\%202013.pdf

3. World Health Organization. Use of liquid TB culture and drug susceptibility testing (DST) in low and medium income countries: Summary report of the expert group meeting on the use of liquid culture media. Geneva, Switzerland: WHO2007. Available from: http://www.who.int/tb/laboratory/use_of_l iquid_tb_culture_summary_report.pdf

4. World Health Organization. The WHO/IUATLD Global Project on Anti- tuberculosis Drug Resistance Surveillance. Anti- tuberculosis Drug Resistance in the World. Fourth Global Report. Geneva, Switzerland. Availableat:Http://www.who.int/tb/publica tions/2008/drs_report4_26feb08.pdf

5. Hasegawa, N., Miura, T., Ishii, K., Yamaguchi, K., Lindner, T.H., Merritt, S., et al., (2002) New Simple and Rapid Test for Culture Confirmation of Mycobacterium tuberculosis Complex: A Multicenter Study. Journal of Clinical Microbiology, 40, 908-912. http://dx.doi.org/10.1128/JCM.40.3.908912.2002

6. Park, M.Y., Kim, Y.J., Hwang, S.H., Kim, H.H., Lee, E.Y., Jeong, S.H., et al., (2009) Evaluation of an Immunochromatographic Assay Kit for Rapid Identification of Mycobacterium tuberculosis Complex in Clinical Isolates. Journal of Clinical Microbiology, 47, 481-484.

7. Tortoli E, Cichero P, Piersimoni C, Simonetti MT, Gesu G, et al., (1999) Use of BACTEC MGIT 960 for recovery of mycobacteria from clinical specimens: multicenter study. J Clin Microbiol 37: 3578-3582.

8. Abe C, Hirano K, Tomiyama T. Simple and rapid identification of the Mycobacterium tuberculosis complex by immunochromatographic assay using antiMPB64 monoclonal antibodies. J
ClinMicrobiol 1999; 37: 3693-7.

9. Maurya AK, Nag VL, Kant S, Kushwaha RA, Kumar M,Mishra V, et al., Evaluation of an immunochromatographic test for discrimination between Mycobacterium tuberculosis complex and non tuberculous mycobacteria in clinical isolates from extra-pulmonary tuberculosis. Indian $\mathbf{J}$ Med Res., 2012; 135: 901-6.

10. Vadwai V, Sadani M, Sable R, Chavan A, Balan K, Naik A, et al., Immunochromatographic assays for detection of Mycobacterium tuberculosis: What is the perfect time to test? Diagn Microbiol Infect Dis 2012; 74: 282-7.

11. Fraser Wares, Balasubramanian R, Mohan A Sharma SK. Extra pulmonary tuberculosis, management and control. In: Agarwal SP, Chauhan LS, editors. Tuberculosis Control in India. New Delhi: Elsevier; 2005:95-114.

12. Correlation of Sputum Gram's Stain and Culture in Lower Respiratory Tract Infections Anuradha Mokkapati, Madhavi Yalamanchili. IOSR Journal of Dental and Medical Sciences (IOSR-JDMS), 8, 6-9.

13. Kanade S, Nataraj G, Suryawanshi R, Mehta P. Utility of MPT 64 antigen detection assay for rapid characterization of mycobacteriain a resource constrained setting. Indian J Tuberc 2012;59:92-6

14. Vadwai V, Sadani M, Sable R, Chavan A, Balan K, Naik A, et al., Immunochromatographic assays for detection of Mycobacterium tuberculosis: What is the perfect time to test? Diagn Microbiol Infect Dis 2012; 74: 282-7.

15. Kumar VG, Urs TA, Ranganath RR. MPT 64 Antigen detection for Rapid confirmation of $\mathrm{M}$. tuberculosis isolates. BMC Res Notes 2011; 24: 79.

16. G Kandhakumari, S Stephen et al., Extra pulmonary tuberculosis: Rapid identification of Mycobacterium tuberculosis grown in Mycobacterium growth indicator tube 960 and 
Lowenstein-Jensen media, employing Standard diagnostics Bioline Mycobacterium tuberculosis protein 64antigen detection kit. Indian Journal of Medical Microbiology, (2015) 33(Supplement 1): S122-25.

17.Petrini, B. and Hoffner, S. (1999) DrugResistant and Multidrug-Resistant Tubercle Bacilli. International Journal of Antimicrobial Agents, 13, 93-97

18.Pillay, M. and Sturm, A.W. (2007) Evolution of the Extensively DrugResistant F15/LAM4/KZN Strain of Mycobacterium tuberculosis in KwaZuluNatal, South Africa. Clinical Infectious Diseases, 45, 1409-1414.

19. Tahaoğlu, K., Törün, T., Sevim, T., et al., (2001) The Treatment of MultidrugResistant Tuberculosis in Turkey. The New England Journal of Medicine, 19, 170-174

20.Sevim, T., Aksoy, E., Ataç, G., et al., (2002) Treatment Adherence of 717 Patients with Tuberculosis in a Social Security System Hospital in Istanbul, Turkey. The International Journal of Tuberculosis and Lung Disease, 6, 25-31.

21.Gupta, S., Bandyopadhyay, D., Gupta, S., Sadhukhan, S. and Banerjee, S. (2012) A Sociodemographic Study of Multidrug Resistant Tuberculosis Cases from DOTS Clinics of Kolkata. Journal of the Indian Medical Association, 110, 723-725.

22. Almeida, D., Rodigues, C., Udwadia, Z.F., Lalvani, A., Gothi, G.D., Mehta, P., et al.,
(2003) Incidence of Multidrug-Resistant Tuberculosis in Urban and Rural India and Implications for Prevention. Clinical Infectious Diseases, 36, 152-154.

23. Ramachandran R, Nalini $S$, Chandershekhar V, Dave PV, Sanghvi AS, Wares F, et al., Surveillance of drug resistant tuberculosis in the state of Gujrat, India. Int J Tuberc Lung Dis 2009; 134: 1154-60.

24. Parmasivan CN, Rehman F, Wares F, Sundar Mohan N, Sundar S, Devi S, et al., First and second line drug resistance patterns among previously treated tuberculosis patients in India. Int J Tuberc Lung Dis 2010; 14: 243-6.

25.Zignol M, van Gemert W, Falzon D, Sismanidis C, Glaziou P, Floyda K, et al., Surveillance of anti-tuberculosis drug resistance in the world: An updated analysis 2007-2010. BullWorld Health Organ 2012; 90: 111-9D.

26. WHO (2008) Anti-Tuberculosis Drug Resistance in the World. Guidelines for the Programmatic Management of Drug Resistant Tuberculosis. World Health Organization, Geneva.

27. Ndung'u, P.W., Kariuki, S., Ng'ang'a, Z. and Revathi, G. (2012) Resistance Patterns of Mycobacterium tuberculosis Isolates from Pulmonary Tuberculosis Patients in Nairobi. The Journal of Infection in Developing Countries, 6, 33-39.

\section{How to cite this article:}

Qursheed Sultana, Ajaz Hussain, Mohammed Abdur Rab Ansari, Mohd Khaleel and Maimoona Mustafa. 2019. Rapid Identification of Mycobacterium tuberculosis and Non Tuberculous Mycobacterium Isolates from Pulmonary and Extra Pulmonary Samples using MGIT320 Liquid Culture System and MPT64 Antigen Test. Int.J.Curr.Microbiol.App.Sci. 8(01): 1172-1182. doi: https://doi.org/10.20546/ijcmas.2019.801.123 